\title{
The surgery of a giant chest wall tumor with intrathoracic extension
}

\author{
Isaac Okyere ${ }^{1}$, Perditer Okyere ${ }^{2}$, Samuel Gyasi Brenu³ ${ }^{3}$ Emmanuel Ameyaw ${ }^{4}$ \\ ${ }^{1}$ Kwame Nkrumah University of Science and Technology, Kumasi, Ghana \\ ${ }^{2}$ Department of Medicine, School of Medicine and Dentistry, College of Health Sciences, Kwame Nkrumah University \\ of Science, Kumasi, Ghana \\ ${ }^{3}$ Department of Surgery, Komfo Anokye Teaching Hospital, Kumasi, Ghana \\ ${ }^{4}$ Department of Child Health, School of Medicine and Dentistry, College of Health Sciences, Kwame Nkrumah University \\ of Science and Technology, Kumasi, Ghana \\ Kardiochirurgia i Torakochirurgia Polska 2021; 18 (2): 113-116
}

The chest or thoracic cavity is a space that is enclosed by the spine, ribs and sternum and is separated from the abdomen below by the diaphragm. The chest cavity contains the heart, the thoracic aorta, lungs, and esophagus. Like any other structure, the chest wall can develop tumors. Desmoid tumors of the chest wall are uncommon fibromatosis tumors characterized by local invasion and frequent recurrence. They are considered low-grade malignant sarcomas. We share our experience with the successful management of a young man who presented with a giant chest wall tumor with extensive intrathoracic extension involving the esophagus, the trachea, the right bronchus, the heart and the great vessels. He underwent successful complete excision with the histopathological examination reporting of aggressive fibromatosis.

A 35-year-old man was referred from a peripheral hospital to our facility on account of a year's history of a progressively increasing right anterior chest wall swelling which initially began in the right axilla and subsequently grew to involve the upper half of the right anterior chest. It was associated with progressive exertional dyspnoea, cough, chest pain, dysphagia to solids and weight loss. There was no prior history of trauma or surgery at the affected site. He resorted to herbal medications for the management of the condition but symptoms did not improve and so subsequently reported for further management especially when he started developing exertional dyspnea. On review by the cardiothoracic surgery team, he looked hemodynamically stable but was dyspneic and orthopneic on room air with a respiratory rate of 24 cycles per minute and saturating at $95 \%$ on room air. There was a hard, lobulated and well-defined mass on the right anterior chest wall measuring $20 \mathrm{~cm} \times 15 \mathrm{~cm}$ in the greatest diameter. It was fixed to the underlying chest wall but not the overlying skin. There were palpable ipsilateral axillary lymph nodes with the largest measuring $1 \mathrm{~cm} \times 1 \mathrm{~cm}$. Air entry was absent on the right hemithorax.
Chest X-ray showed homogeneous opacification of the right hemithorax in the typical white-out syndrome phenomenon with contralateral mediastinal shift. A computed tomography chest scan revealed a large heterogeneously enhancing mass filling the right hemithorax measuring $20.5 \mathrm{~cm} \times 13.3 \mathrm{~cm}$ and extending to the right anterior chest wall with chest wall extension measuring $12.7 \mathrm{~cm} \times 10.9 \mathrm{~cm}$. The mass was compressing and displacing the trachea to the left with a contralateral displacement of the heart and the great vessels. The contralateral lung was normal with no lytic or sclerotic bony lesions observed. All hematological, biochemical and serological investigations done showed normal parameters. Due to the extensiveness of the mass and its associated symptomatology, the patient was counselled for a staged excision. The first stage involved the excision of the anterior chest wall component and the second stage which was done after 1 week involved the excision of the intrathoracic tumor and the freeing of the right lung and the adherent intrathoracic structures. The second stage tumor resection was done under a double-lumen endotracheal anaesthesia with the patient being positioned in a left lateral decubitus position. A standard right posterolateral thoracotomy was done entering the $6^{\text {th }}$ intercostal space. The findings included a huge, well-encapsulated, yellowish, firm, smooth-surfaced intrathoracic tumor involving the chest wall and covering the whole right thoracic cavity with total collapse of the right lung. The tumor was carefully dissected from its surrounding structures and excised by careful blunt and sharp dissection as shown in Figure 1. It weighed $6 \mathrm{~kg}$. The lung air leaks were repaired with vicryl 4/0 and the chest was closed up in layers after hemostasis with warm normal saline irrigation was done, leaving a size $32 \mathrm{Fr}$ chest tube in the chest. The patient was extubated in the operating theatre. Postoperative recovery was gradual and complicated by formation of seroma at the surgical site which was evacuated. He also developed right

Address for correspondence: Isaac Okyere MD, FWACS, FGCS, Kwame Nkrumah University of Science and Technology, Kumasi, Ghana,

e-mail: isaac.okyere@knust.edu.gh

Received: 19.01.2021, accepted: 14.03.2021. 


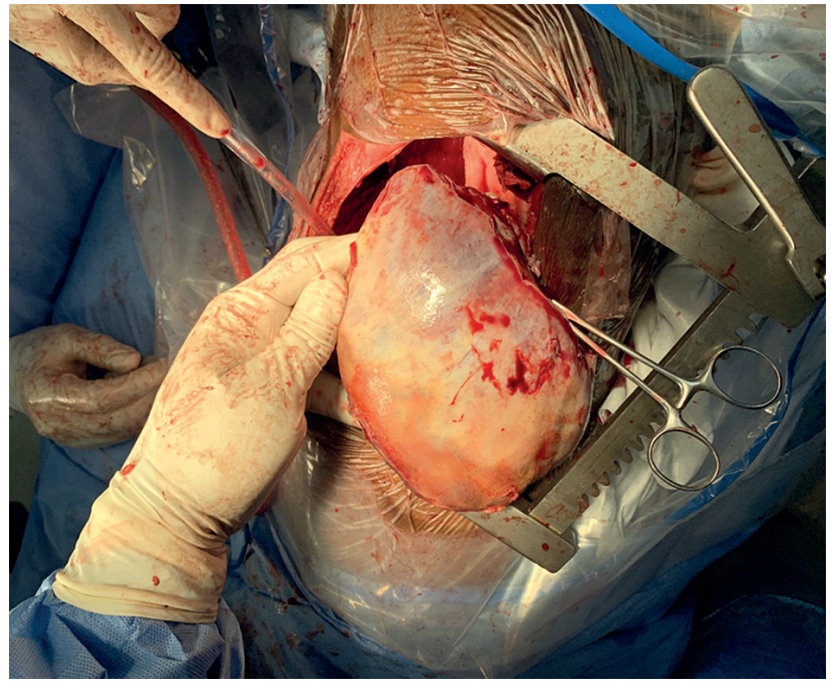

Figure 1. Intraoperative photograph of the delivery of the intrathoracic giant monster tumor

pleural effusion which was drained by needle thoracocentesis. He was eventually discharged home on postoperative day 22 for outpatient follow-up. Repeat chest X-rays done on out-patient follow-up as shown in Figure 2 showed an adequately expanded right lung. Histopathological analysis of the specimen showed spindle cells with fibroblast like nuclear features and areas of thick collagen. The lesion was infiltrating between skeletal muscle tissues. The features were consistent with aggressive fibromatosis. Two years after surgery, there is a small recurrence at the apex of the right hemithorax for which the patient has been referred to the oncologist for further evaluation. Otherwise, he looks very healthy.

Desmoid tumors are rare soft tissue tumors of fascial or musculoaponeurotic origin which are found to account for $3.5 \%$ of all fibrous tumors, $0.3 \%$ of all solid tumors and less than $0.03 \%$ of all tumors [1-4] and with a reported incidence of 2-4 cases per million of population [1]. Since their initial description in 1832 by John MacFarlane [5], various terminologies - such as desmoid fibroma, desmoma, desmoplastic fibroma, aponeurosis fibromatosis, aggressive fibromatosis or sarcoma of low-grade malignancy - have been used to describe it $[2,5]$. Aggressive fibromatosis or sarcoma of low-grade malignancy is the currently preferred terminology due to its locally infiltrative potential and risk of recurrence despite radical excision [3-5]. However, their inability to metastasize distinguishes them from fibrosarcomas $[3,6]$. Our case was consistent with aggressive fibromatosis as was reported by the pathologist. Desmoid tumors generally occur within the ages of 15-60 years [3], although a similar chest wall tumor was reported by Govindarajan in a 7-year-old boy [7]. They have been found to have a predilection for females, with a $3: 1$ female-to-male ratio $[1,2]$. This, along with their regularity among women of childbearing age increased the incidence with oral contraceptive use and their regression beyond menopause or with use of anti-estrogens has led to the suggestion that

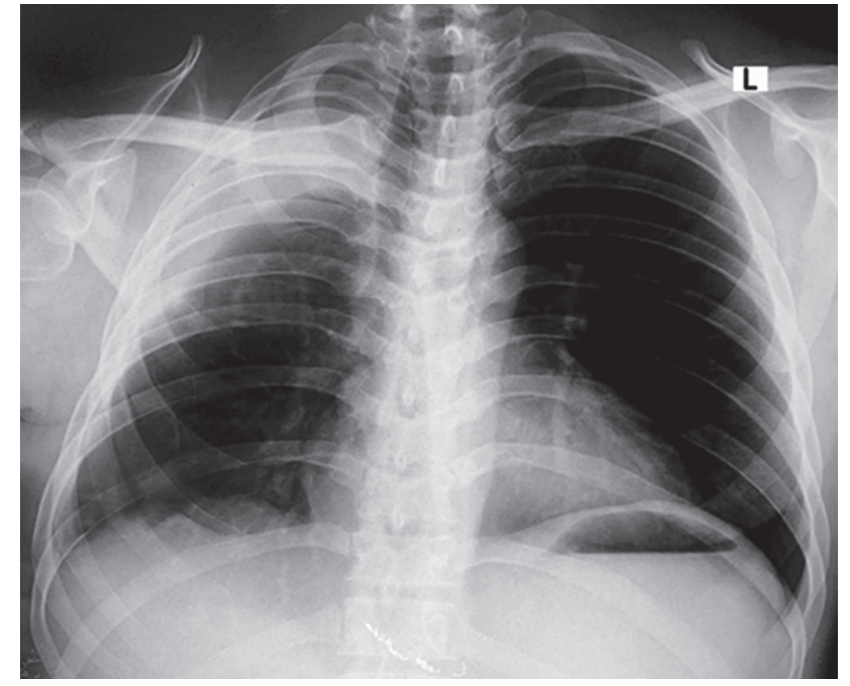

Figure 2. Postoperative chest $\mathrm{X}$-ray at 9 months

sex hormones may be involved in the pathogenesis of desmoid tumors [1]. Other risk factors reported include genetic predisposition or history of previous trauma [2, 3, 5]. Our patient was a 35-year-old man, consistent with the common age group at increased risk.

Based on the anatomical localization of the tumor, aggressive fibromatosis can be classified as extra-abdominal, abdominal or intra-abdominal [1]. About half of all desmoid tumors have been found to occur in the abdominal region [7]. Common extra-abdominal sites of desmoid tumors reported in the literature include the upper and lower extremities, the head and neck, and the chest wall [2, 5], with our case being an example of an extra-abdominal type involving the chest wall and the intrathoracic cavity, as shown in Figure 3. The chest wall is the most common site of extra-abdominal fibromatosis with a reported incidence of $20 \%$, but reports of true intrathoracic aggressive fibromatosis as in our case are very rare $[3,5]$. Intrathoracic desmoid tumors originate from the pleura or mediastinum and are mainly located within the thoracic skeleton with some chest wall involvement $[1,3]$. They are thought to originate from the pleura or subpleural mesenchymal cells [1].

Usually, desmoid tumors of the abdominal wall, thoracic wall and extremities are relatively small because they often present as palpable masses which are picked up early. Intrathoracic desmoid tumors, on the other hand, are usually not diagnosed until they are big enough to cause mass effects on surrounding vital structures [2]. Our case presented with a huge anterior chest wall mass with total white-out syndrome of the right hemithorax signifying a total occupancy of the right hemithorax causing compressive effects on the intrathoracic structures, namely, the esophagus, trachea, right main bronchus, the heart and the great vessels, leading to total collapse of the right lung. Similar to our case, Matrai and associates reported that $60 \%$ of the reported cases of intrathoracic aggressive desmoid tumors measure more than $10 \mathrm{~cm}$ in diameter [1]. 


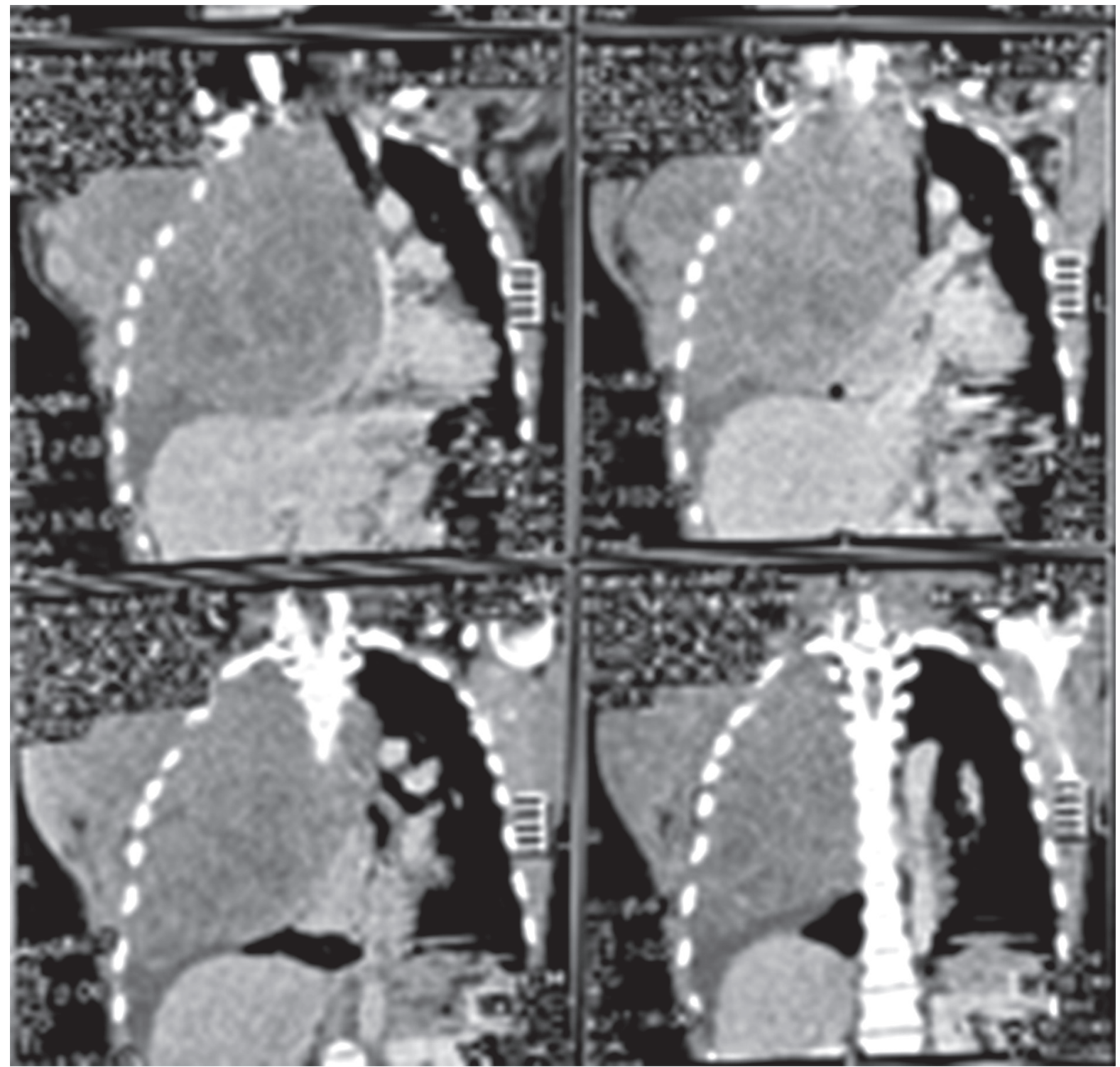

Figure 3. Preoperative CT chest scan showing the giant tumor occupying the whole right hemithorax and the anterior chest wall

Like all other giant intrathoracic tumors, common presenting symptoms include dyspnea on exertion or dyspnea due to tracheobronchial or vagal compression, cough and dysphagia $[1,8]$. They may also cause pain and discomfort from the mass effect or paresthesia and muscle weakness from nerve entrapment [6]. Symptoms have been found to precede diagnosis by about 12-16 months [1]. Our patient presented with symptoms and signs of respiratory obstruction, dysphagia to solids, dyspnea and orthopnea after a year of noticing the growing mass.

Chest radiography in intrathoracic desmoid tumors may only present as an opacity in the affected hemithorax [1] as was classically demonstrated in the CT chest scan shown in Figure 3. CT scans and MR imaging provide further detail into the size, location and involvement of surrounding structures. It may be of variable density relative to the surrounding soft tissues with no necrosis, calcifications or hemorrhage $[1-3,6]$. Our patient was evaluated using a primary screening imaging study, chest X-ray, which showed the huge white-out syndrome or opacity with shift of the mediastinum to the contralateral side, and this was further delineated using the CT scan of the chest as shown in Figure 3. Histopathological evaluation is invaluable in the establishment of diagnosis. Microscopically, there are myofibroblasts without microscopic features of malignant potential in a collagenous background [2]. Our histopathology report confirmed the diagnosis of aggressive fibromatosis.

The mainstay of management of intrathoracic desmoid tumors is radical tumor resection with negative tumor margins $[1,3,4,9,10]$. In the event of surgical excision not being possible, neoadjuvant radiotherapy may be employed to reduce tumor size or to aim for a possible cure [3]. Adjuvant radiotherapy may also be employed as its been seen to reduce recurrence rates [3, 9]. However, it should be used with caution particularly in the young due to concerns of tissue fibrosis and radiation-induced cancers [3]. Other 
treatment modalities such as anti-estrogen therapy have also been reported $[3,6]$. Our patient had radical resection and did not receive any adjuvant radiotherapy and did well for two and a half years before the recurrence.

Rates of recurrence have been quoted to range from $29 \%$ to $54 \%$ according to Alexander in 2014 [3], but a rate as high as $60 \%$ was reported by Shah et al. [6]. Recurrence has been found to occur mostly within 2 years after excision [3], and therefore regular follow-up is mandated even in tumor-free resections $[3,6]$.

In conclusion, complete surgical resection of huge chest wall tumors with intrathoracic extension is potentially curative and therefore it should be the modality of choice for early diagnosis of chest wall tumors especially in less resourced centers.

\section{Acknowledgments}

Written informed consent was obtained from a relative of the patient for the publication and accompanying images.

\section{Disclosure}

The authors report no conflict of interest.

\section{References}

1. Mátrai Z, Tóth L, Szentirmay Z, Papp J, Langmár Z, Kásler M. Multidisciplinary treatment of intra-thoracic desmoid tumors: Case series and narrative review [Internet]. Vol. 18, Medical Science Monitor. International Scientific Literature Inc.; 2012 [cited 2020 Jun 28]. p. CS17. Available from: /pmc/articles/ PMC3560762/?report=abstract

2. Chen JH, Jin EH, Chen GY. A huge intrathoracic fibromatosis with rapid aggression. Chin Med J 2012; 125: 4530-2.

3. Alexander GR. Largest recorded noninvasive true intrathoracic desmoid tumour. South African J Surg 2014; 52: 114-5.

4. Yörük Y, Karamustafao lu YA, Sezer YA, Ibiş AC. Extra-Abdominal aggressive fibromatosis presenting as an intrathoracic tumor. Trak Univ Tip Fak Derg 2010; 27: 302-4.

5. Ibrahim M, Sandogji H, Allam A. Huge intrathoracic desmoid tumor. Ann Thorac Med 2009; 4: 146-8.

6. Shah J, Prasad S, Patkar D, Kothari S. Intrathoracic extension of aggressive fibromatoses. Br J Radiol 2000; 73: 66-8.

7. Govindarajan KK. Chest wall fibrolipoma presenting as a massive dumbbell tumour in a child. Kardiochir Torakochir Pol 2020; 17: 170-1.

8. Aggarwal D, Dalal U, Mohapatra PR, Singhal N. Intra-thoracic desmoid tumor. Lung India 2012; 29: 160-2.

9. Hagmaier RM, Nelson GA, Daniels LJ, Riker Al. Successful removal of a giant intrathoracic lipoma: a case report and review of the literature. Cases I 2008; 1: 87.

10. Bolke E, Krasniqi H, Lammering G, Engers R, Matuschek C, Gripp S, Gerber PA, Fischer G, Peiper M, Shaikh S, Budach W, Orth K. Chest wall and intrathoracic desmoid tumors: surgical experience and review of the literature. Eur J Med Res 2009; 14: 240-3. 